\title{
User Interfaces for Geometric Modeling
}

\author{
M. Shpitalni, H. Lıpson \\ ${ }^{I}$ CMSR Laboratory for Computer Graphics and CAD \\ Department of Mechanical Engineering \\ Technion-Israel Institute of Technology \\ Haifa, Israel 32000 \\ Email: shefi@tx.technion.ac.il \\ ${ }^{2}$ Mechanical Engineering Department \\ Massachusetts Institute of Technology \\ Cambridge MA 02139-4307, USA \\ Email: hlipson@mit.edu
}

\begin{abstract}
The aim of this paper is to focus attention on the role of the user interface in the field of geometric modeling. The need to handle, perceive and manipulate spatial information in three dimensions and related difficulties as well make the potential benefits of user interfaces particularly attractive. We review some of the recent related work and indicate where future challenges and opportunities may lie.
\end{abstract}

Key words: $\quad \mathrm{CAD}$, Geometric Modeling, User Interface

\section{INTRODUCTION}

At a recent conference, several well-known scientists assembled in attempt to predict the most important factors that will impact computer research and applications in the next fifty years (Beyond computation, 1997). While history has proven that such attempts are but mere speculation, it is interesting to note that the human-computer interface was brought up as one of the central prospective issues. One essay, for example, compares the human-computer interface to the architecture of a building. While the structure of a building supports its functionality, it is the architecture that determines the space in which the user lives and works; this space can either promote or inhibit productivity, restrict or encourage exploration. Indeed, the user interface is often the single most important determining factor in the 
success or failure of an interactive system or application (Baecker et al, 1995). Gibbs (1997), Nielsen (1993) and Bias (1994) document numerous cases in which attention to quality of an interface, or lack of it, have directly affected cost, reliability and productivity benefits. Moreover, recent surveys of commercial systems (Bobrow et al, 1986; Myers and Rosson, 1992) indicate that 30 to 50 percent of application code and comparable amounts of development time are devoted to aspects of user interfaces. Yet despite these indications, the human-computer interface remains one of the most poorly understood elements of systems. In particular, research in geometric modeling has overlooked this aspect for a long time.

In its basic definition, a user interface comprises the input and output mechanism and underlying software that drive the interaction with the user. In a broader sense, the user interface can be said to include anything that shapes the user's experience in achieving a particular task via computer (Baecker et al, 1995). In light of this definition, this paper aims to focus on the role of the user interface in the geometric modeling field. The need to handle, perceive and manipulate spatial information in three dimensions and related difficulties as well make the potential benefits of user interfaces particularly attractive. In this paper, we review some of the recent related work and attempt to indicate where future challenges and opportunities may lie.

Interaction with spatial geometric models opens a multiplicity of possible modes of interaction. Some of these modes deal directly with object manipulation, while other deal with display of related information or inference of user intent.

In this paper we skip over the description of the traditional 'graphic user interface', along with related issues such as simple direct manipulation of three dimensional objects, zooming, panning and rotation, and various discourse methods such as menus and dialogs. These methods are applied today to almost any information technology field, and are therefore beyond the scope of this paper. For a historical perspective on the development of these methods see (Baecker et al, 1995). The following sections elaborate on more recent approaches and demonstrate how they can be employed in geometric modeling tasks. Some examples from a working implementation in our lab are demonstrated.

\section{MANIPULATIVE USER INTERFACES}

User expectation is often based on real world practice and feedback, particularly in geometric object manipulation that is strongly analogous to daily experience. There has therefore been increasing interest in seamlessly 
blending physically manipulative objects with corresponding virtual artifacts. When the user manipulates the physical object, the anticipated effects are applied to the virtual counterpart. This approach greatly reduces the effort associated with learning a traditional manipulation interface, and makes manipulation more fluent and intuitive.

Manipulative user interfaces can be applied to many object parameters, from simple translation and rotation to more complex operations such as squeezing, sliding, and tilting. In many cases, the physically manipulated object can be related to a particular task; for example, a hinge with a digital angle-gauge has been used as a physical control for adjusting the angle of a joint in a geometric model. Fitzmaurice et al (1995) use artificial "bricks" as a graspable user interface. Ishii et al (1997) describe "phicons" to bridge manipulation and tactile feedback between users and microscopic or abstract geometry, such as atoms and bits. Harrison et al (1998) describes a tactile user interface that allows direct squeezing/sliding and tilting. This is achieved using an elongated pressure/position strip along the boundaries of the display. Sliding a finger along the display boundary produces a squeezing/sliding effect intuitively similar to that created by flipping a book page by squeezing/sliding along its edge. Similarly, static pressure can produce a grabbing/holding effect, and tilting of the display produces a tilting/rotation effect of the corresponding digital element.

\section{CONCEPT METAPHORS}

In contrast to the hardware metaphors discussed in the previous section, concept metaphors (or design metaphors) are exploited by designers to create an analogy between a common daily concept and a particular computerized task. The metaphor is intended to help the user discover and understand properties of the target functionality by simple analogy. Common design metaphors include viewing the screen as a desktop, considering the delete functionality as a trash can, or seeing programs as agents. For example, the trashcan metaphor enables us to conceive of the undelete operation as a retrieval of objects from the trash, as opposed to the permanent erasure associated with emptying the trashcan. Designers and users usually re-interpret properties of the source when transferring to the target. For instance, when working with a desktop we select an object instead of grasping it, or place a file on top of a printer to print rather than physically stacking one on top of the other. Carrol et al (1988) and Erickson (1990) discuss the foundations for understanding and working with design metaphors. 
Recently, design metaphors have been applied to more specific tasks such as geometric modeling. One example is Stubblefield's work (1998) on using a spell-checker metaphor to design a 'Machinability Advisor' based on geometric considerations. The point is to conceive of unnecessarily tight tolerances, features that require specialized machine tools, failure to standardize features across a part and difficult machining geometries - as language errors in a text document. This metaphor was used to create an intuitive tool for scanning such errors and correcting them; it offers a framework for comparison with other operations, such as replacing repetitive occurrences, ignoring certain situations or adding them to a customized library, as well as obtaining 'suggestions' for correction of common errors.

\section{PHYSICALLY BASED INTERACTION AND NAVIGATION}

For many years, the tacit assumption of geometric interfaces was that geometric specification would be explicit, accurate and unambiguous. As geometric modeling moves from mere calculation to more abstract and creative design, there is a growing need to support simple 'free-hand' manipulation of 'free-form' shapes. Although such shapes typically require a complex mathematical representation, their manipulation must retain simplicity, and match the qualitative experience of manipulating free-form materials in real life (e.g. clay).

To achieve this kind of interface, it is necessary to have some means of simulating the physical behavior of plastic and elastic materials, force fields and resistance. The simulation need not be perfectly accurate, but must be sufficient to provide realistic feedback. In particular, much work has concentrated on evaluating simplified models for behavior of surfaces under applied forces and under various constraints of curvature, slope and position. Using these systems, a user can poke, pull and deform surfaces and boundary-representation solids and get a realistic result. However, recent work also has also addressed guiding issues of using physical simulation to help users interact and move within complex geometric environments, where traditional manipulation/navigation techniques are insufficient (Xiao and Hubbold, 1998).

\section{USER ATTENTION}

While most traditional geometric interfaces rely on icons and menus to activate commands and to retrieve information, this approach contradicts the 
growing trend to use pure and realistic graphical representations. In an attempt to preserve realism, such representations shun handles, labels and other gadgets that would prompt the user for interaction. Instead, the concept of 'hot spots' has been introduced. Hot spots are locations on the objects that have a certain functionality or uniqueness; they are generally invisible, but when a graphic user approaches them, they may provide some feedback, by changing the shape of the cursor, for example.

While the concept of 'hot spots' has been used primarily in the context of virtual reality and web-navigation, this idea is also being used in geometric modeling as a means to decrease clutter in display of information as well as to display or obtain information in context of a local area and a specific operation. For example, we naturally expect hot spots at boundaries and corners of resizable objects. In a geometric scene, hot spots can exist at any location of a unique geometric property, such as an endpoint, an edge, a midpoint, an intersection point or a center point. More complex spots may be introduced at mass centroids, or at locations that are likely to induce some particular operation. For example, in a model of a mechanism, hot spots may be introduced to allow varying a particular degree of freedom.

The use of hot-spots provides an opportunity for embedding functionality and information within a geometric model without overwhelming the user with unneeded possibilities. If hot-spots are presented consistently, experienced users learn to expect them at certain locations. However, the almost infinite possibilities for interaction and the fact that these spots are initially invisible may create problems; hence their placement and arrangement is a subject of research in itself. Plante et al (1998) and Milanese et al (1994) describe ways to anticipate zones more likely to attract attention, based on such properties as color, geometry and feature discontinuity.

\section{MULTIMODAL COMMUNICATION AND VISUAL TRACKING}

While direct manipulation and traditional interfaces rely on specially tailored devices, multimodal methods use natural modes of communications such as speech, gestures, gaze, finger/hand-pointing and facial expressions. Although these modes of communication are indirect, they are considered effortless and intuitive for humans. They present an opportunity for fluent and efficient communication, but at the same time present a severe real-time computational and algorithmic challenge. The challenges are multiple; first, the communicating human must be tracked and singled out from a possibly noisy background. Then, communicated messages must be captured and 
filtered. Finally, the captured message must be interpreted, often in context of the current operation, of other messages and of previous messages, and in context of the user's history. For practical reasons, current implementations of multimodal communications are typically limited to well-defined tasks. The multimodal approach is particularly attractive for communication in virtual or augmented environments, where the use of devices may be cumbersome.

Several works address issues related to tracking facial features and expressions in real time for multimodal interaction (see, for example, Yang et al (1998)) under various noise, lighting and background conditions. Cipolla and Hollinghurst (1998) describe a system for real-time uncalibrated identification of finger pointing. They apply their method to direct robot motion, but many other geometric applications come to mind. Di Bernardo et al (1998) discuss methods for monocular tracking of the human arm in three dimensions. Beyond capturing gestures, the human arm can also serve to define spatial form and to activate mechanisms.

Capturing gestures is a particularly difficult task if the gestures are to be interpreted as having a symbolic meaning. In a geometric modeling context, a hand wave may be used to indicate that an object is to move away or come closer or spin, or that its characteristics may be queried. Yachida and Iwai (1998) and Cassel (1998) address some of these issues.

Figure 1 illustrates the basic setup of a gesture capturing system we have constructed (Meermeier, 1998). A camera is set up so that it has a clear view of the user workspace. A fiducial color target is mounted on the user's hand. The target is chosen so that it will not interfere with routine activity of the user, such as typing or using the mouse. Two such targets are shown in Figure 2. A real-time vision algorithm is then executed to track the position of the target. A comparison of the projection of the target on the camera with the original (known) geometry can determine the current spatial location and orientation of the hand with respect to the camera. This information is then used to reflect the hand motions onto an object; Figure 3 shows how a user using this system manipulates a teapot object in the background.

An additional aspect of multimodal user interfaces is output. In the same way that users may convey information easily using natural communication, users can also accept information more easily if it is conveyed in a natural form. For example, Cassel's work (1998) also addresses the issue of generating gestures to provide natural feedback. Munch and Dillmann (1998) discuss ways to create haptic output; their system applies force feedback to the mouse so that users inadvertently feel resistance or release when they approach a certain location on an object or an interface gadget. This conveys a sense of rejection or acceptance, without any explicit communication. 


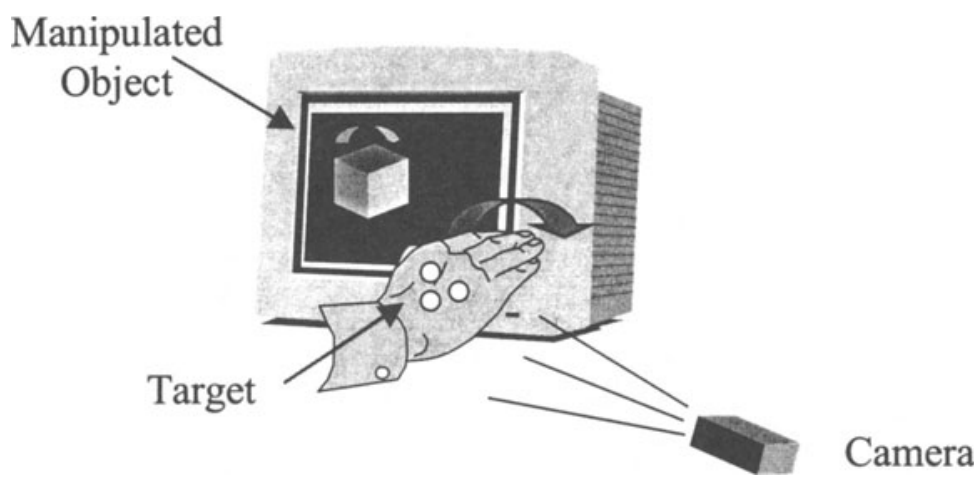

Figure 1. Basic setup for capturing hand gestures for object manipulation.
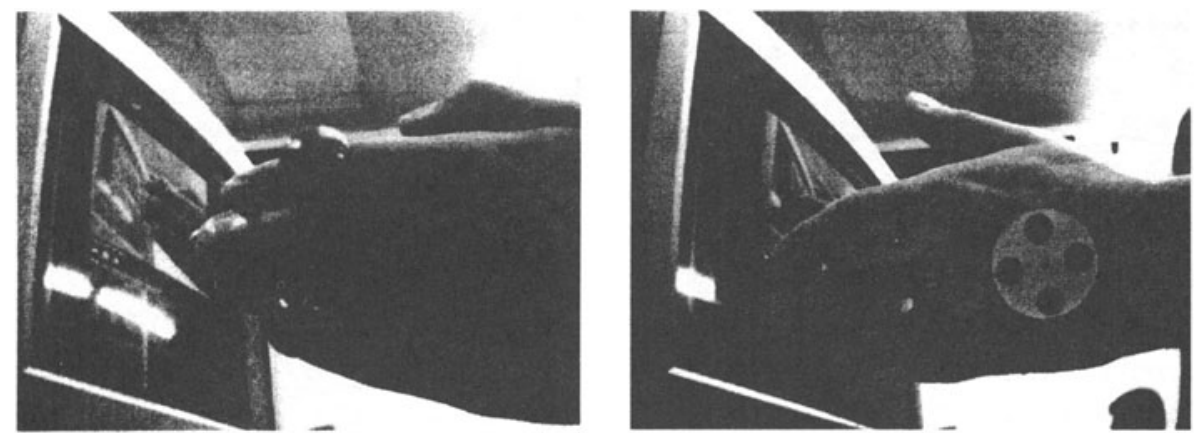

Figure 2. Two hand-mounted targets for visual tracking.
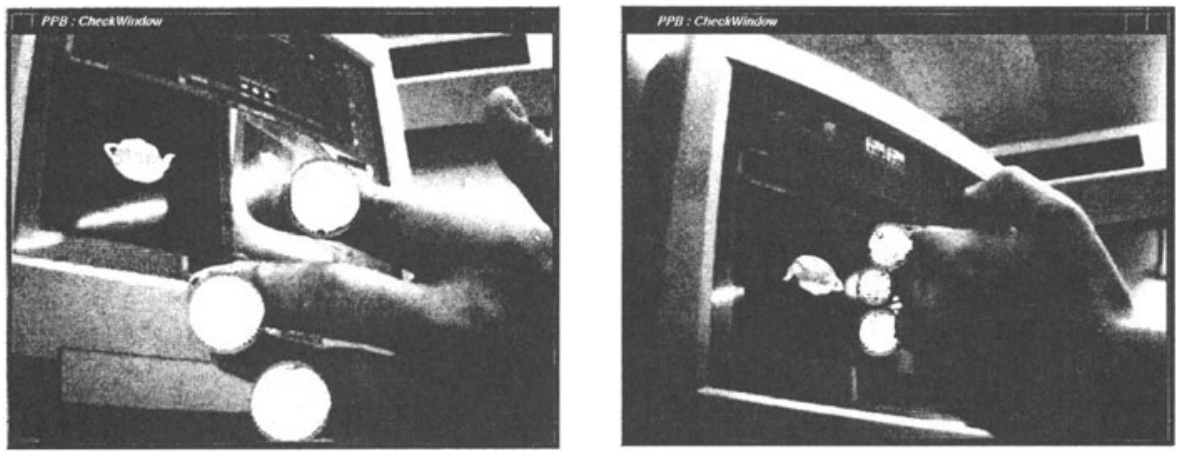

Figure 3. Manipulating a teapot using hand motion. 


\section{AUGMENTED AND VIRTUAL ENVIRONMENTS}

Just a few years ago, virtual and augmented reality were no more than a game. In recent years, however, it has become apparent that these forms hold the capacity to become a valuable engineering tool as well. Beyond realistic "walk-throughs" and "fly-overs", there is a trend towards applying these technologies to industrial applications, geometric modeling being but one possibility. Virtual reality (VR) is characterized by real-time simulation and presentation of virtual three-dimensional worlds, set up so that the user can be totally (or nearly totally) immersed within the environment, and interact with it. Dai (1998) describes a multitude of applications of virtual reality to industrial environments, including virtual prototyping, manipulation and simulation of virtual objects. In particular, aspects pertaining to physical simulation are addressed, such as realistic dynamic and contact simulation and ergonomic issues.

After almost a decade, however, the inherent limitations of VR have become apparent Although technical drawbacks such as image blur, update rate and response lag are bound to improve as technology progresses, a more inherent drawback of VR is that the user is cut off from the immediate surroundings, so that most of the simple activities of any daily working environment are precluded. Despite the groundbreaking potential of immersive VR, the fact that during a VR session it is almost impossible to jot something down in a notebook, pick up a phone, interact with someone who just entered the room or even see one's own body diminishes its usefulness as an everyday tool.

When applying augmented reality techniques, much less information is displayed by the computer. Hence, the updating rate can be significantly increased so that a better response is achieved. Head-up displays are combined with immersive VR so that users can see the natural environment overlaid with virtual augmentations at specific points. This method eliminates the need to model the environment, and less information must be generated by the computer. Stereoscopic views with appropriate position dependent visual cues can be used to create the illusion of visual augmentation while maintaining contact and allowing dynamic interaction with the surroundings.

Figure 4 shows two implementations of augmented reality in engineering applications. Figure 4(a) shows a maintenance application where maintenance instructions are overlaid onto the maintained device using a geometric/information model of the instrument. Figure 4(b) shows a view of a milling tool overlaid with synthetic dimensions, as seen by the operator. Further information can be found in (Lipson and Shpitalni, 1998). 


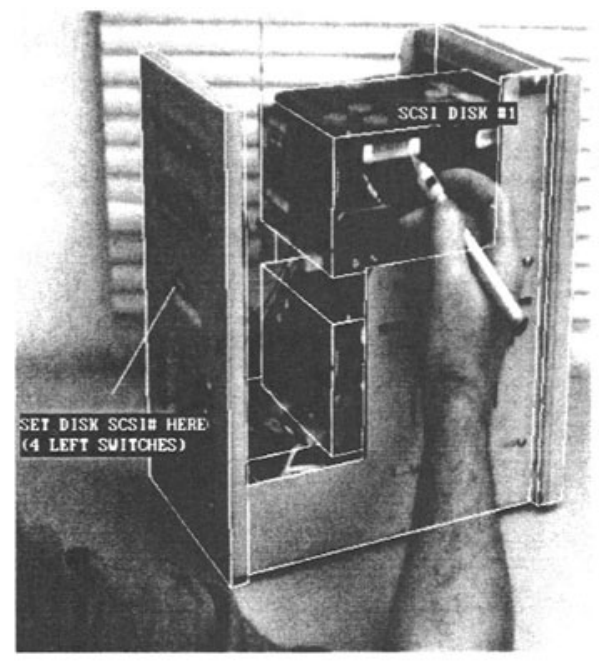

(a)

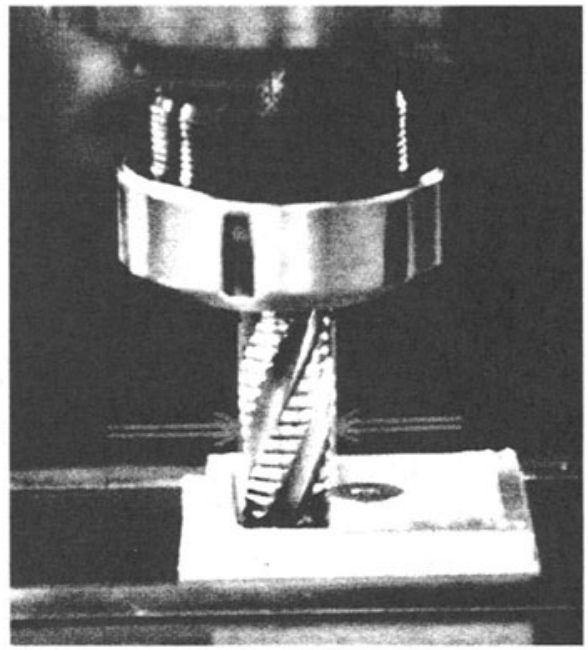

(b)

Figure 4. Reducing information load using augmented reality: (a) in maintenance, instructions are overlaid onto the maintained device using non-immersive augmented reality, (b) a view of a milling machine tool overlaid with a synthetic dimension, as seen by the operator, in an immersive augmented reality setup.

\section{SKETCHING}

It is interesting to watch how an engineer, when given a design problem, instinctively reaches for a pencil and paper. The importance of drawing, both formal drafting and informal sketching, has been the subject of extensive research. In a series of experiments, Ullman et al (1990) show the necessity of drawing during all developmental stages of a mechanical design. Ullman notes that "... engineers are notorious for not being able to think without making "back-of-the-envelope" sketches of rough ideas ... Sometimes these informal sketches serve to communicate a concept to a colleague, but more often they just help the idea take shape". In a study of architectural design, Herbet (1987) defines sketches as "informal, private drawings that architectural designers use as a medium for graphic thinking in the exploratory stages of their work". According to Herbert's theory, sketches provide an extended memory of the visual images in the designer's mind, and, since they can be made rapidly, allow for more facile manipulation of ideas at various levels of abstraction. In a survey of the adequacy of CAD tools for conceptual design (Puttre, 1993), the author emphasizes the fluency, flexibility and inaccuracy of sketches as a primary quality. An 
industrial designer, relating to an existing CAD system, is quoted as saying "The interface is just not for us. I can do thirty sketches on paper by the time it takes me to do two on the computer". In summary, sketching appears to be important for the following reasons:

1. It is fast, suitable for the capacity of short term memory

2. It is implicit, i.e. describes form without a particular structure

3. It serves for analysis, completeness check and simulation

4. It is inexact and abstract, avoiding the need to provide unnecessary details

5. It requires little commitment, is easy to discard and start anew

Despite the fact that a good proportion of an engineer's representation is informal sketching, CAD systems today do not support sketching in any meaningful way. In a recent work carried out at our lab, we attempted to investigate the possibilities for a design environment suitable for the conceptual stage of product development (Lipson, 1998). The emphasis is on allowing the designer and the system to communicate rough geometric information by sketching spatial concepts.
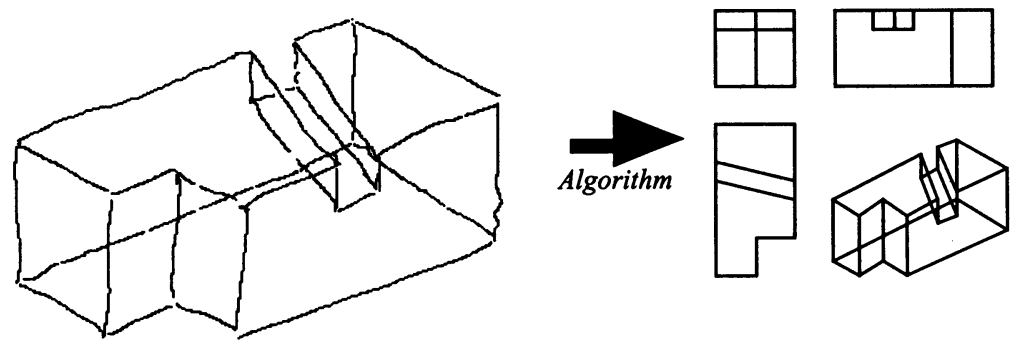

Figure 5. Conceptual design by sketching - an illustration.

Figure 5 above illustrates a key constituent of the proposed approach. On the left we see a freehand sketch, the kind of sketch one draws on the back of an envelope in a few seconds. We would like the computer to be able to "look" at this sketch, just like an engineer colleague would, and perceive the three-dimensional object (product) depicted in it. Once the object has been perceived, the computer can proceed to analyze and reason about this product, predict its key properties and perhaps its manufacturing problems, based on the sketch alone, just like a human expert. In the future, the computer may also provide alternative designs. Furthermore, once depth of the object has been reconstructed, it is possible to change the orientation of the depicted object in three dimensions, and continue sketching more details from a different viewpoint. In this respect, freehand sketching using this approach surpasses the possibilities of sketching with a pencil on paper, 
where the viewpoint is fixed and cannot be changed. Once the object is complete, it may be transformed into an accurate model. However, since sketching is an implicit mode of specification, no construction scheme exists. Consequently, the transition from a rough to an accurate threedimensional model must be achieved using techniques such as variational geometry.
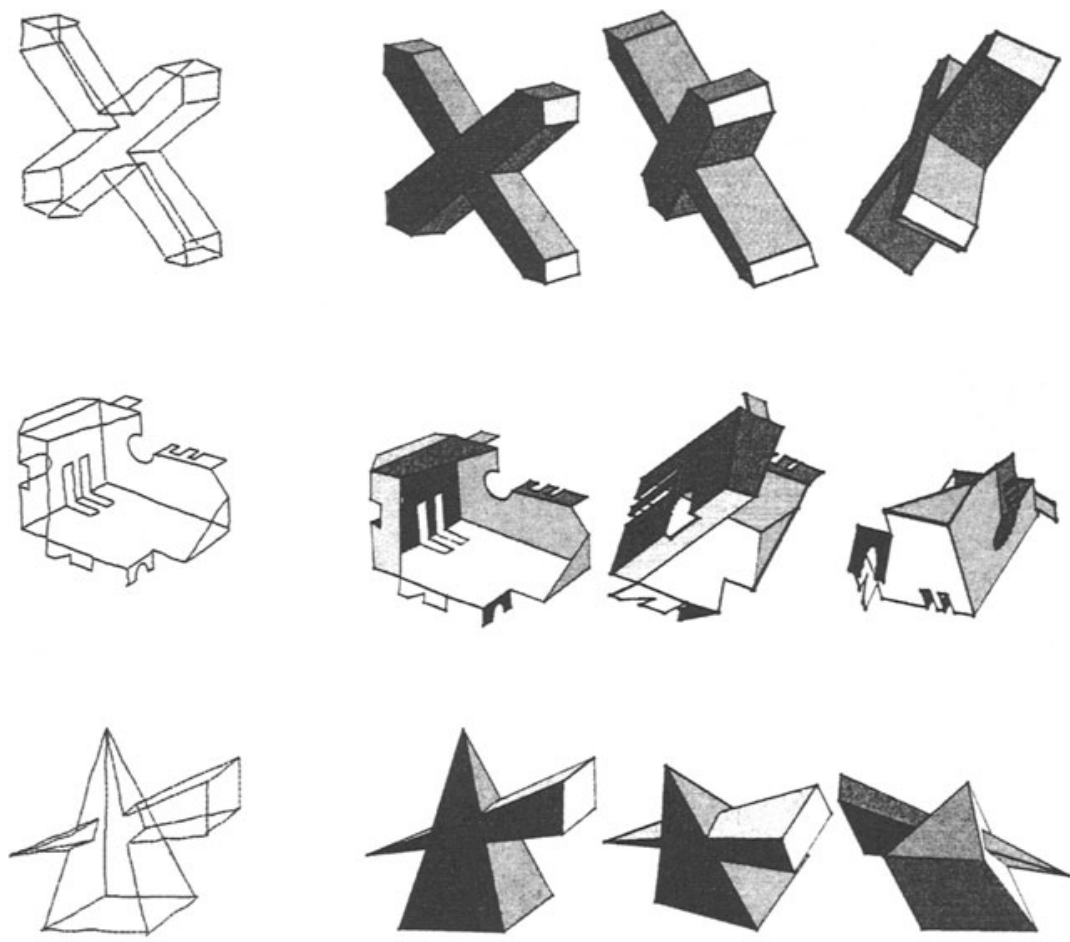

(a)

(b)

Figure 6. (a) product sketches, (b) the reconstructed 3D models.

Although restoration of a three-dimensional object from its projection is a mathematically indeterminate step, it can be achieved using image cues. Figure 6 above presents some sample results. This technique was incorporated into a system for conceptual design of sheet metal products. The system accepts a sketch of the product, reconstructs its spatial form and creates an engineering model to be analyzed. The results of the analysis are conveyed to the user by displaying them back on the sketch. The results contain numeric data, drawings of possible flat patterns, and illustrations of selected bend lines, as well as error estimators for some of the results. Although these results can be expressed in an organized and 'neat' output 
format, an attempt has been made to convey the results in context of their original specification, so as to make them more easily accessible. This has been achieved by (a) highlighting bend lines as rough marks overlaid on top of the original sketch using the reverse projection which was applied for reconstruction, and (b) displaying the output flat patterns using rough synthesized sketch strokes, with amplitude corresponding to overlap error, in order to convey the notion that the results are not accurate and to indicate the expected uncertainty. Figure 7 below illustrates an analysis sequence.

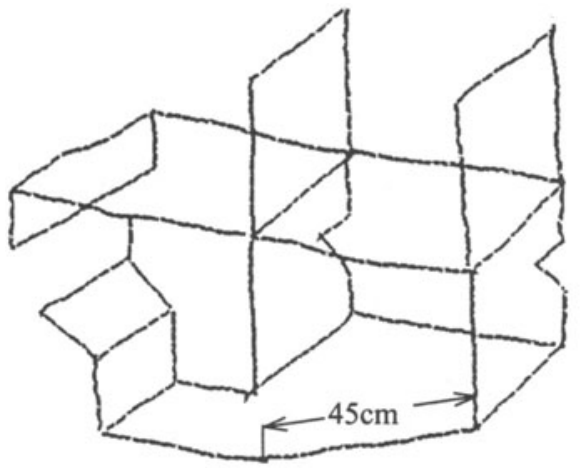

(a)

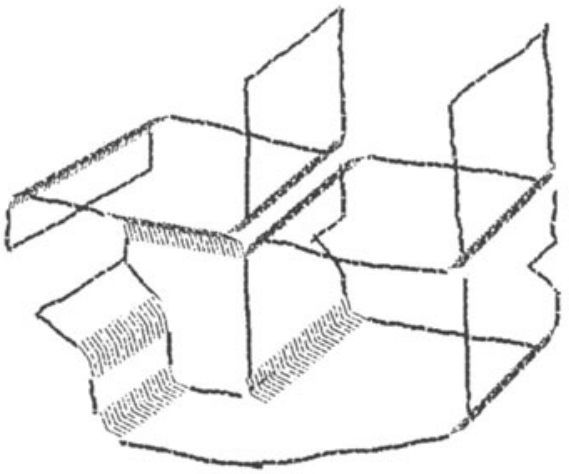

(c)

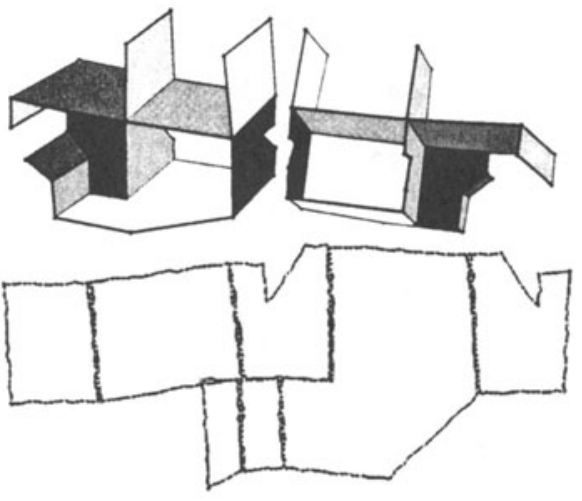

(b)

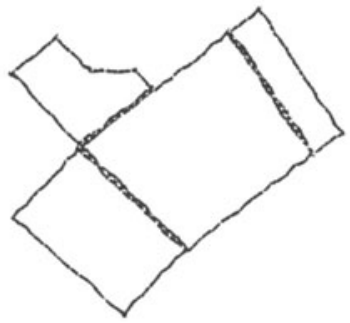

Material: Steel Thickness: $3[\mathrm{~mm}]$

Area: $3.27\left[\mathrm{~m}^{\wedge} 2\right]$ Weight: $39[\mathrm{Kg}]$

Components: 2 Nesting eff: $61 \%$

Raw mat: $63[\mathrm{~kg}]$ Weld length: $3.4[\mathrm{~m}]$ Bends: 9

Cost: 1018.38

Figure 7. (a) original flat (2D) sketch, (b) reconstructed 3D model, (c) optimal bend assignments overlaid on original sketch, and (d) optimal flat pattern and predicted product properties

The hardware interaction device, shown in Figure 8 below, provides a natural environment for freehand interaction using a virtual drawing table that supports natural pen-and-paper-like interactive sketching. This apparatus supports both input and output on the same real sheet of paper. 


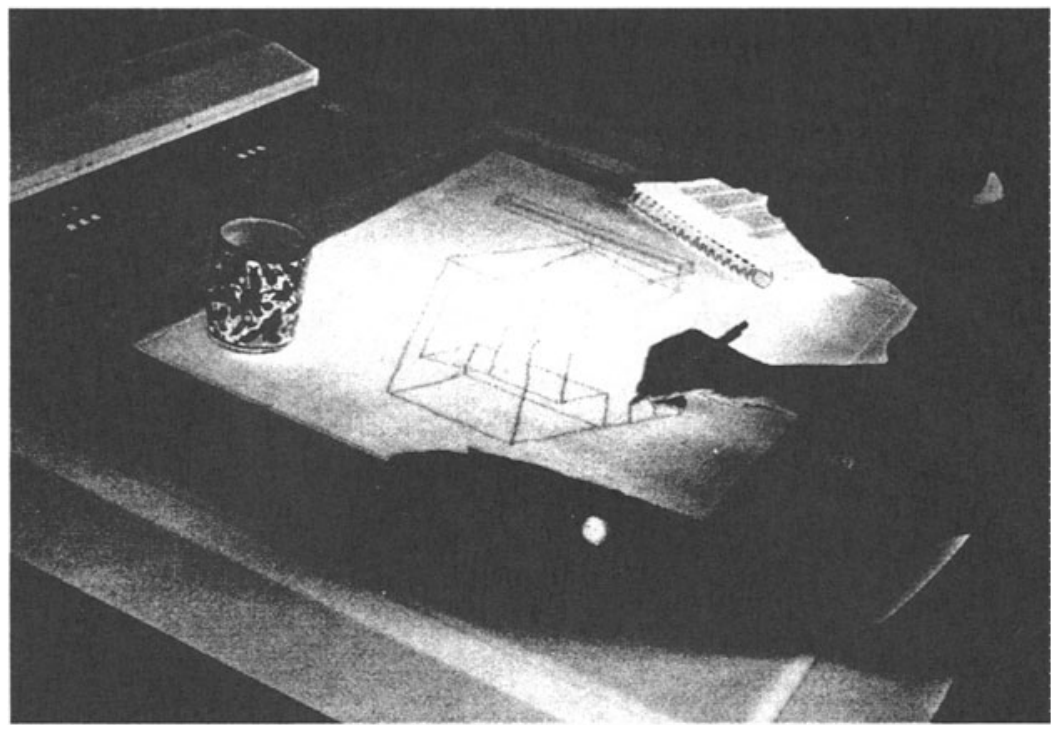

Figure 8. Virtual pen-and-paper-like sketching environment.

\section{USER MODELING}

According to the user-modeling paradigm, the productivity of user interfaces can be enhanced if applications construct and maintain computational models of each user's ability, knowledge, skills and goals. This goes beyond simple maintenance of user preferences, as most programs do, because the user model can then be employed to diagnose errors or misconceptions, chart progress or offer guidance and advice, tailored for a particular user and circumstances. Traditionally, user modeling has employed symbolic schemes to represent a user's knowledge state. Recently, however, this concept has been expanded to permit modeling cognitive abilities such as spatial cognition; this information directly relates to the ability of users to effectively perceive and manipulate three-dimensional scenes. Eisenberg et al (1998) discuss an educational system called hypergami, in which students design three-dimensional polyhedra. The system is used to learn, adapt and determine what makes particular threedimensional structures and orientation more or less difficult to visualize for a particular user. This information can later be used to enhance his or her productivity.

Other modes of user modeling can be introduced. A system may discover typical work patterns associated with a particular user, preferred viewpoints 
and common operation sequences. This information may then be used by agents and wizards to anticipate and automate tasks.

\section{AGENTS AND WIZARDS}

Computers are usually conceived of as passive tools, yielding information upon request. This passiveness constitutes a major drawback, because it depletes one of our most limited resources: attention. Traditional direct-manipulation $\mathrm{CAD}$ interfaces alone are not sufficient. Future geometric modeling software will not merely respond to user requests but, based on previous activities and built-in knowledge, will anticipate user needs and actively seek ways to support the user, exhibiting autonomy and intelligence. Software tools exhibiting these qualities are often termed 'agents'. Several examples follow:

Automated alternative seeking. Given a particular engineering solution, the computer may search 'in the background' for alternative solutions that can provide the same functionality under the same constraints; once these alternatives are found, they are presented to the user. Even if the alternative is not satisfactory, it may stimulate the user to think in new directions. This capability requires automatic derivation of functionality and constraints, as well as automatic solution. The need to seek a variation on an existing solution rather than a completely new solution makes this capability feasible. Some examples include: replacing a part in a solid assembly with a lighter component that has the same function; identifying a new relevant component via the Internet; or verifying the correctness of analysis results and suggesting improvements.

Automated presentation of relevant information. Instead of presenting the user with all available information, the system should be selective and self-initiate presentation of the relevant information that is appropriate at that instance. Examples include automatic selection of the best orientation of a CAD model for working on a particular area of the model; automatic display of the dimensions appropriate for a particular machining task; or automatic online guided maintenance (Lipson and Shpitalni, 1998).

\section{CONCLUSIONS}

Interaction with spatial geometric models opens a multiplicity of possible modes of interaction. In this paper we review some of the recent approaches. Some of these deal directly with the manipulation of objects, while others deal with display of related information or inference of user intent. In this 
paper we attempted to focus attention on the role of the user interface in the geometric modeling field, where the need to handle, perceive and manipulate spatial information in three dimensions spawns many challenges and opportunities.

\section{REFERENCES}

1. Baecker R., Grudin J., Buxton W. A. S., Greenberg S., 1995, Human computer interaction: Towards the year 2000, Morgan Kaufmann, USA

2. Bias R., Mayhew D., 1994, Cost justifying usability, Academic Press

3. Borrow D., Mittal S., Stefnik M., 1986, "Expert systems: peril and promise", Communications of the ACM, Vol. 29 No. 9, pp. 880-894

4. Carroll J., Mack R., Kellog W., 1988, "Interface metaphors and user interface design", in Hellander (Ed.), Handbook of Human Computer Interaction, Elsevier: Amsterdam

5. Cassel J., 1998, “A framework for Gesture generation and interpretation", in Cipolla R. and Pentland A. (Eds.), Computer vision for human-machine interaction, Cambridge university press, Cambridge, pp. 191-216

6. Cipolla R., Hollinghurst N. J., 1998, "A human-robot interface using pointing with uncalibrated stereo vision", in Cipolla R. and Pentland A. (Eds.), Computer vision for human-machine interaction, Cambridge university press, Cambridge, pp. 97-110

7. Dai F. (Ed.), 1998, Virtual reality for industrial applications, Springer verlag, Berlin

8. Di Bernardo E., Goncalves L., Perona P., 1998, "Monocular tracking of the human arm in 3D", in Cipolla R. and Pentland A. (Eds.), Computer vision for human-machine interaction, Cambridge university press, Cambridge, pp. 155-170

9. Eisenberg M., Nishioka A., Schreiner M. E., 1998, "Helping users think in three dimensions: Step towards incorporating spatial cognition in user modeling", Intelligent User Interfaces IUI'97, pp. 113-120

10. Erikson T., 1990, "Working with interface metaphors", in Laurel B. (Ed.) The art of human-computer interface, Addison-Wesley: Reading, Mass.

11. Fitzmaurice G., Ishii H., Buxton W. A. S., 1995, "laying the foundations for graspable user interfaces", Proceedings of CHI'95, pp. $422-449$

12. Harrison B. L., Fishkin K. P., Gujar A., Mochon C, Want R., 1998, "Squeeze me, hold me, tilt me! An exploration of manipulative user interfaces", Proceedings of CHI'98, pp. 17-24

13. Ishii H., Ullmer B., 1997, "Tangible bits: towards seamless interfaces between people, bits and atoms", Proceedings of CHI'97, pp. 234-241

14. Meermeier R., 1998, "Gesture based manipulation of CAD objects", an internal report, Mechanical Engineering, Technion - Israel Institute of Technology

15. Milanese R., Wechsler H., Gil S., Bost J. M., Pun T., 1994, "Integration of bottom up and top down cues for visual attention using non-linear relaxation", Proc. Computer Vision and Pattern Recognition, pp. 781-785

16. Munch S., Dillmann R., 1998, "Haptic output in multimodal user interfaces", Intelligent User Interfaces IUI'97, pp. 105-112

17. Myers B., Rosson M., 1992, "Sirvey of user interface programming". CHI'92, ACM, pp. 195-202

18. Nielsen J., 1993, Usability engineering, Academic press.

19. Plante A., Tanaka S., Inoue S., 1998, "Evaluating the location of Hot-Spots in interactive scenes using the 3R toolbox", Proceedings of CHI'98, pp. 117-123 
20. Stubblefield, W. A., 1998, "patterns of change in design metaphor: A case study", Proceedings of CHI'98, pp. 73-80

21. Xiao D. and Dongbo R., 1998, "Navigation guided by artificial force fields", Proceedings of CHI'98, pp. 179-186

22. Yachida M., Iwai Y., 1998, "Looking at gestures", in Cipolla R. and Pentland A. (Eds.), Computer vision for human-machine interaction, Cambridge University Press, Cambridge, pp. 292-311

23. Yang J., Stiefelhagen R., Meier U., Waibel A., 1998, "Visual tracking for multimodal human computer interaction", Proceedings of CHI'98, pp. 140-147 